\title{
Schwannoma in the porta hepatis - laparoscopic excision under laparoscopic ultrasound guidance
}

\author{
Maciej Sebastian ${ }^{1}$, Maciej Sroczyński ${ }^{1}$, Piotr Donizy ${ }^{2}$, Jerzy Rudnicki ${ }^{1}$ \\ ${ }^{1}$ Department of Minimally Invasive Surgery and Proctology, Wroclaw Medical University, Wroclaw, Poland \\ ${ }^{2}$ Department of Pathomorphology and Oncological Cytology, Wroclaw Medical University, Wroclaw, Poland
}

Videosurgery Miniinv 2017; 12 (3): 301-305

DOI: https://doi.org/10.5114/wiitm.2017.68795

\begin{abstract}
Schwannomas are usually benign tumors attached to peripheral nerves and are rarely found in the gastrointestinal tract. Schwannomas in the porta hepatis are extremely rare, with only 15 cases described in the literature to date. A 22-year-old female patient presented with colicky upper abdominal pain lasting 3 months. Magnetic resonance imaging of the abdominal cavity revealed a tumor in the porta hepatis. The patient was qualified for laparoscopy. The tumor was totally excised laparoscopically under guidance of laparoscopic ultrasound without intra- or postoperative complications. Postoperative histopathological examination confirmed the porta hepatic schwannoma. The patient recovered uneventfully with very good cosmetic results. In the follow-up period of 5 months we have not observed any abdominal or general health problems. The present case is the first report in the world of laparoscopic ultrasound guided laparoscopic excision of a porta hepatic schwannoma.
\end{abstract}

Key words: laparoscopy, schwannoma, ultrasound, magnetic resonance imaging.

\section{Introduction}

Schwannomas are usually solitary peripheral nerve sheath tumors which in most cases appear sporadically, though some are associated with neurofibromatosis type 2, schwannomatosis, Carney's complex or radiation, very rarely undergo malignant transformation and respond well to excision in accessible locations [1]. They rarely occur in the abdominal cavity, particularly the stomach. Treatment of schwannoma in the porta hepatis consists of complete excision with free margins without lymphadenectomy. The overall prognosis is very good [1].

\section{Case report}

A 22-year-old woman was admitted to our department on September 29, 2016 complaining of col- icky upper abdominal pain lasting 3 months. There were no abnormalities found on clinical examination or in the laboratory investigations. The patient did not have any abdominal operations, and family and personal history was without relevance. Abdominal magnetic resonance imaging (MRI) revealed a $3.5 \times$ $2 \times 2 \mathrm{~cm}$ tumor in the porta hepatis without invasion of the vessels or bile ducts (Photos $1 \mathrm{~A}, \mathrm{~B}$ ).

After obtaining informed consent the patient was qualified for laparoscopy. All procedures followed were in accordance with the ethical standards of the 1964 Declaration of Helsinki and its later amendments. The trocar sites were identical as in standard laparoscopic cholecystectomy (we used two $5 \mathrm{~mm}$ trocars and two $10 \mathrm{~mm}$ trocars) - a $10 \mathrm{~mm}$ trocar at the umbilicus (camera), a $10 \mathrm{~mm}$ trocar below the xiphoid process (dissector/grasper/ultrasound probe), a $5 \mathrm{~mm}$ trocar

\footnotetext{
Address for correspondence

Maciej Sebastian MD, PhD, Department of Minimally Invasive Surgery and Proctology, Wroclaw Medical University, 213 Borowska St,

50-556 Wroclaw, Poland, phone: +48 607868 096, e-mail: mseba@op.pl
} 

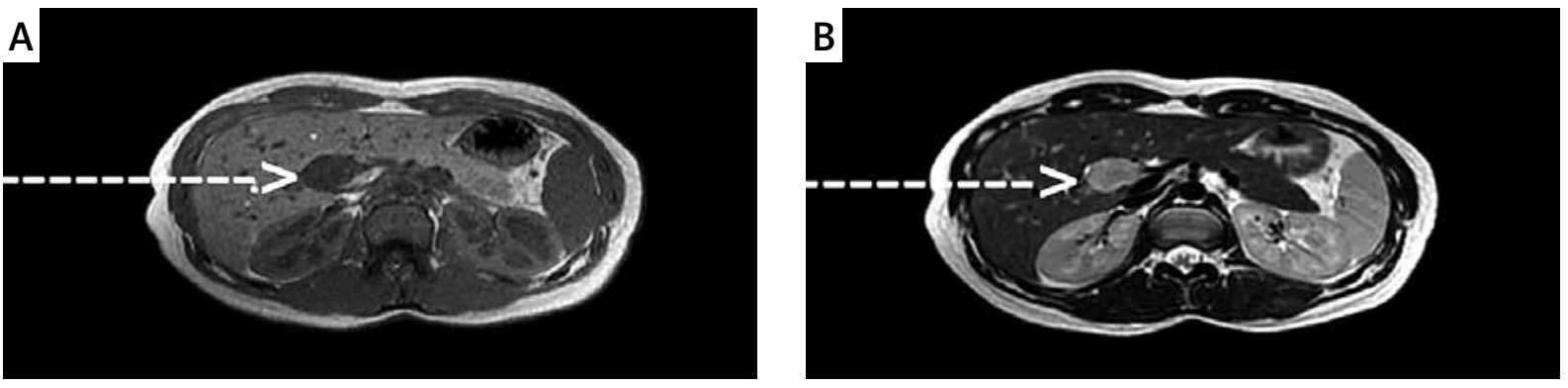

Photo 1. Magnetic resonance imaging revealed a $3.5 \times 2 \times 2 \mathrm{~cm}$ tumor in the porta hepatis without invasion of the vessels and bile ducts. A - Low signal in T1-weighted imaging (arrow). B - High signal intensity in T2-weighted imaging (arrow)
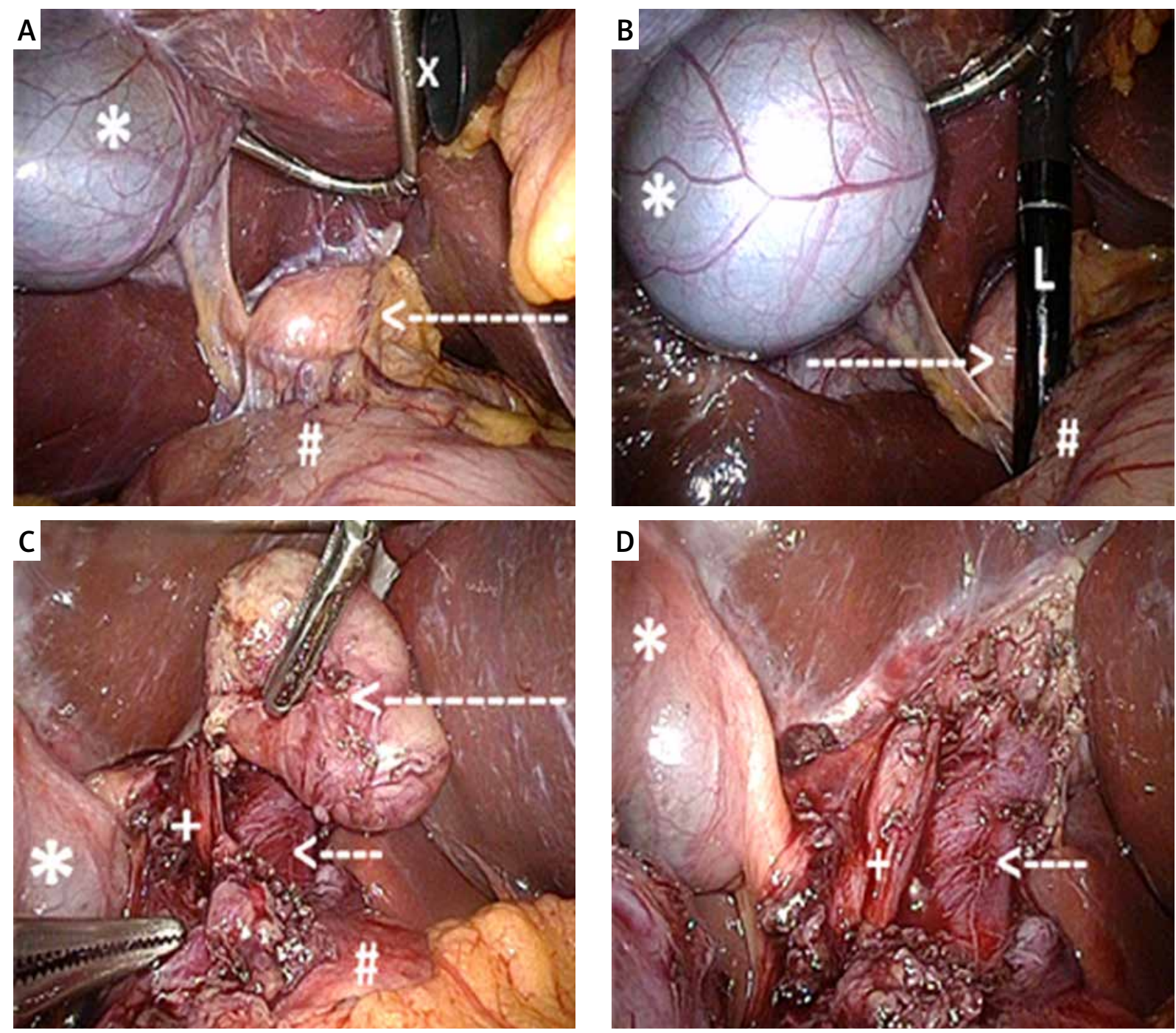

Photo 2. A - Tumor in the porta hepatis (long arrow). Gallbladder ( $*$ ) and duodenum (\#). Liver is retracted with snake liver retractor $(\mathrm{x})$. B - Laparoscopic ultrasound $(\mathrm{L})$ defines the correct plane of dissection. Tumor (long arrow), gallbladder $(*)$ and duodenum (\#). C - Excision of tumor (long arrow) is carried out in healthy margins without injury to the bile duct $(+)$ and portal vein (short arrow). Gallbladder $(*)$ and duodenum (\#). D - Bile duct $(+)$ and portal vein (short arrow) are very clearly visible after excision of tumor. Gallbladder $(*)$ 
below the right costal arch (dissector/grasper) and a $5 \mathrm{~mm}$ trocar in the middle line between the umbilicus and the xiphoid process (retractor). An ultrasound probe was inserted through the epigastric $10 \mathrm{~mm}$ trocar. We used the laparoscopic probe Toshiba PEF-704 LA (frequency 7.0 $\mathrm{MHz}$ ) and the diagnostic ultrasound system Toshiba NemioMX SSA-590A. Snake liver retractor $5 \mathrm{~mm}$ was used for liver traction (Photos 2 A-D). Duplex Doppler function enabled differentiation between the vascular and avascular structures. Laparoscopic ultrasound (LUS) was performed several times during surgery and enabled a safe plane of dissection. It was extremely useful during dissection and separation of the tumor from the bile duct because there was no well-defined plane in this localization (Photos 3 A, B). Intraoperatively and postoperatively we did not observe any complications. Postoperative histopathological and immunohistochemical examination revealed a totally excised $3.5 \times 2 \times 2 \mathrm{~cm}$ benign schwannoma S100 (+), smooth muscle actin (SMA) (-), CD117 (-), CD34 (+) - only in blood vessels, Ki-67 (+) $<1 \%$ of tumor cells (Photos $4 \mathrm{~A}-\mathrm{D}$ ). The patient recovered uneventfully and we did not observe any complications during the 5-month follow-up period.

\section{Discussion}

Porta hepatic schwannomas are extremely rare, with only 15 cases reported in the literature to date [2].
They originate from the sympathetic and parasympathetic fibers which are distributed along blood vessels in this localization. Macroscopically, schwannomas are spheroidal or ovoid in shape. Larger tumors may undergo secondary degeneration [2, 3]. Pathogenesis of both sporadic and genetically acquired schwannomas is associated with defects in the merlin signaling pathways. Differential diagnosis should include gastrointestinal stromal tumors (GIST), leiomyomas, nerve sheath tumors, lymph node tuberculosis, lymphoma, giant lymph node hyperplasia, hemangiomas and Klatskin tumor. Immunohistochemically they are strongly S100 reactive, SMA negative, CD34 negative and CD 117 negative. Leiomyomas are negative for $\mathrm{S} 100$ and positive for SMA. A few gastrointestinal tumors (GIST) are also positive for S-100, but they are also positive for either CD34 or CD117 [4-6].

The mean age of patients in the reported cases was 48 years (29-74 years) and the male-female ratio was $6: 9$. The exact location of the tumor was the porta hepatis ( 9 patients), the hepatoduodenal ligament (5 patients) and the proper hepatic artery (1 patient). The mean tumor diameter was $4.7 \mathrm{~cm}$ $(2.2-7.5 \mathrm{~cm})$. Symptoms were associated with compression of adjacent structures. Nine (60\%) patients were symptomatic and complained of distension/ abdominal discomfort (5 patients - 33\%), abdominal pain (4 patients - 27\%), jaundice/dark urine/
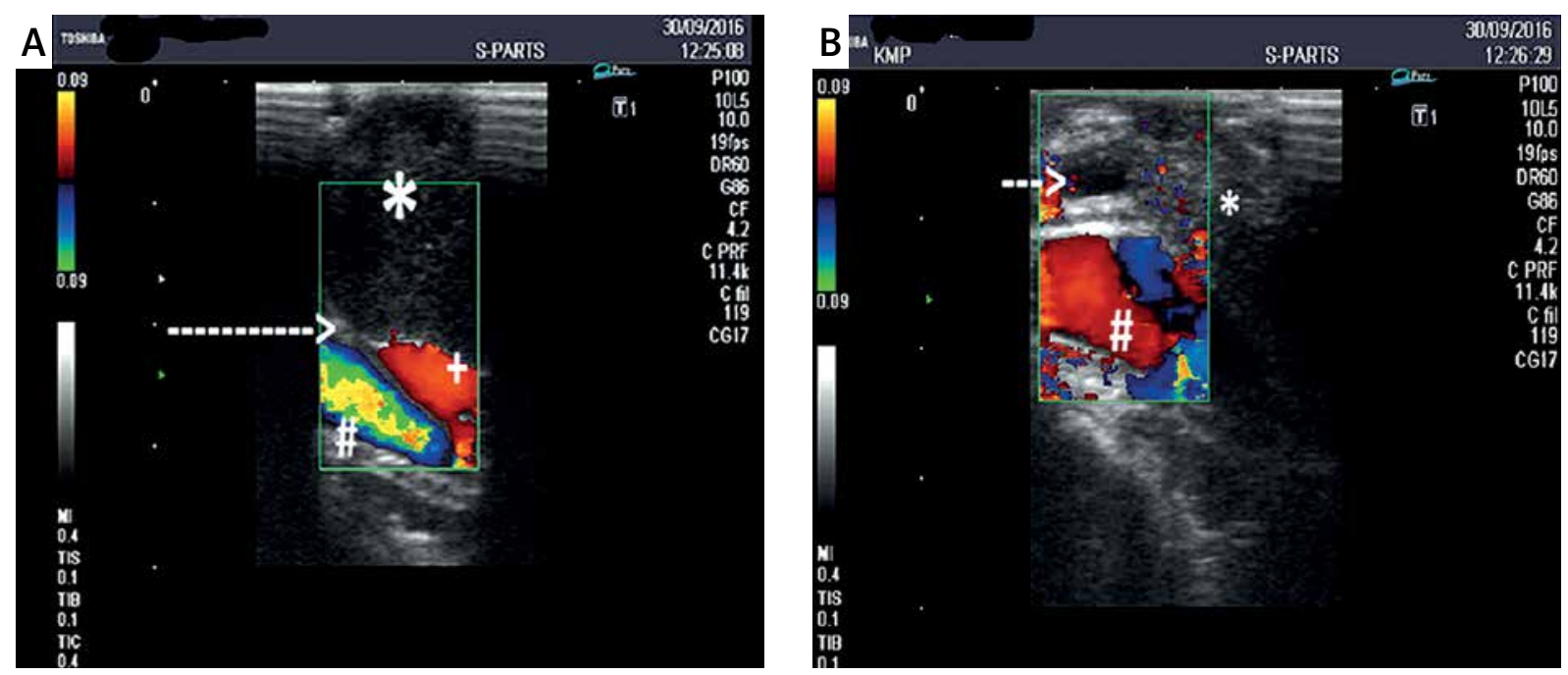

Photo 3. A - Laparoscopic ultrasound revealed a hypoechoic mass $(*)$ lying over the portal vein (\#) and proper hepatic artery (+). Hyperechoic capsule of tumor (long arrow) without angioinvasion enables safe dissection over vessels. B - There is no clearly visible border between the tumor $(*)$ and the bile duct (short arrow) but careful dissection in this plane did not injure the bile duct. Portal vein (\#) 

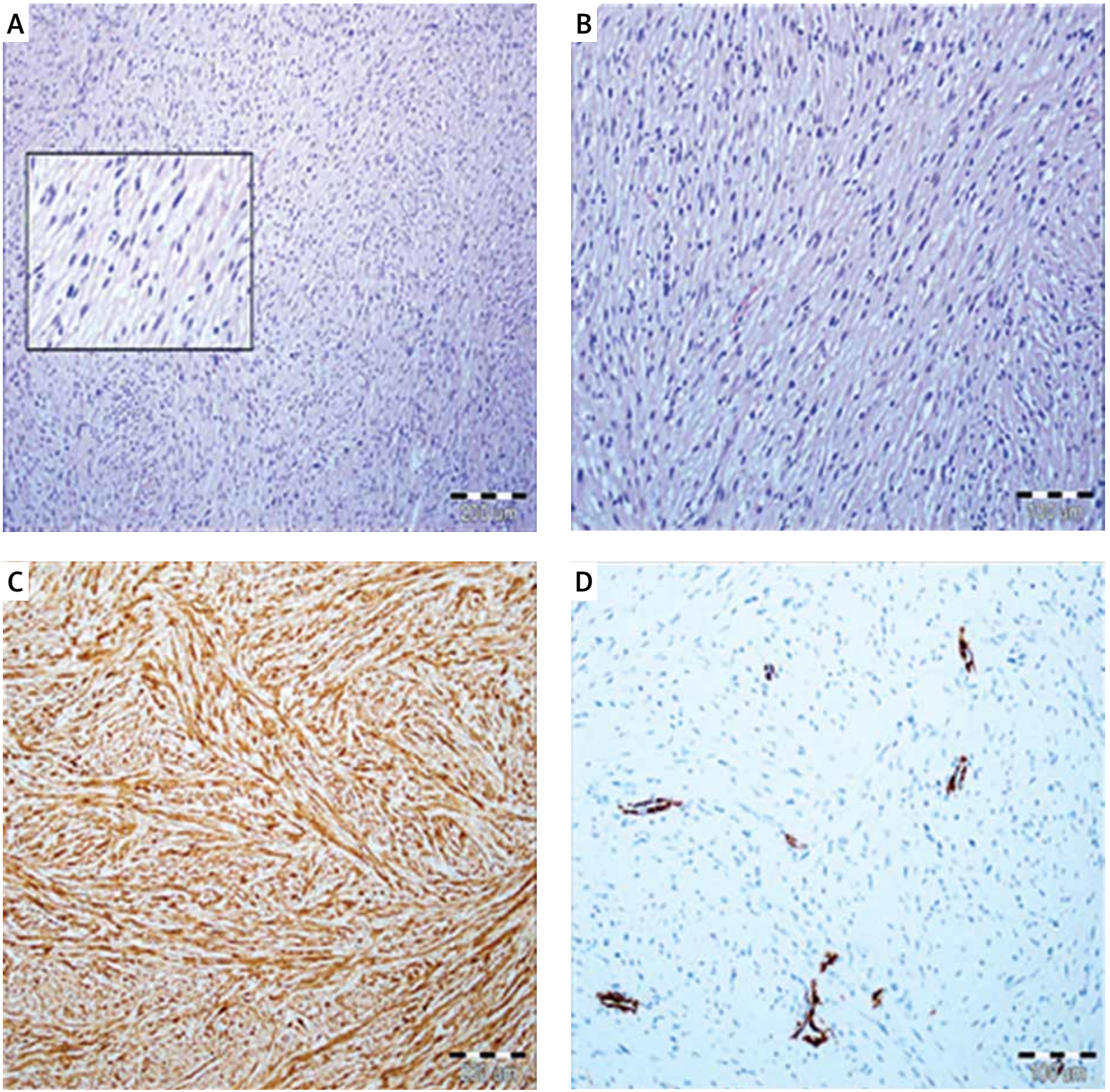

Photo 4. Liver tumor mass with solid proliferation of interlacing fascicles of fusiform cells. Atypical mitotic figures and fields of necrosis were not observed (A): 100x, 400x, H\&E staining; (B): 200x, hematoxylin and eosin (H\&E) staining. Strong S100 protein expression in tumor cells (C): 100x, hematoxylin. Lack of smooth muscle actin (SMA) immunoreactivity in neoplastic cells with strong expression observed only in blood vessels (D): 200x, hematoxylin

colorless stool/pruritus ( 2 patients - 13\%), nausea/ vomiting/anorexia (1 patient $-7 \%$ ) and weight loss (1 patient - 7\%). Preoperative diagnostic imaging was nonspecific. Ultrasound imaging revealed a solid isoechoic or hypoechoic well-bordered mass, computed tomography a heterogeneous mass with peripheral enhancement, MRI a mass with low signal intensity in $\mathrm{T} 1$ and high signal intensity in
T2-weighted imaging [7, 8]. Fine needle aspiration performed preoperatively in 2 patients revealed suspected spindle cell neoplasia or stromal tumor [2].

Only 7 patients out of 15 underwent complete tumor excision. Due to invasion of adjacent structures 1 patient had en bloc right hepatectomy, 2 patients en bloc resection of the hepatic artery, 1 patient partial resection of the extrahepatic bile duct with 
Roux-en-Y hepaticojejunostomy and one patient had a T-tube bile duct drainage due to the bile duct injury. There were no recurrences in the mean follow-up period of 16 months (3-41 months) among 6 postoperatively observed patients. All the operations were performed in an open manner and there were no reports about the use of LUS [2].

The LUS during diagnostic laparoscopy is used in the preoperative staging of pancreatico-biliary cancers for detecting unresectable disease, enabling direct assessment of the peritoneal cavity, liver, lymph nodes and related vascular structures. The LUS is also used for staging of hepatocellular cancer or metastasis of colon cancer, staging of gastric cancer, to localize tumors during adrenalectomy and in biliary disease as an alternative to intraoperative cholangiography to identify choledocholithiasis and anatomical relationships in the plane of dissection [9, 10]. Laparoscopic surgery results in minimal injury to the tissues, smaller post-operative wounds and less post-operative pain, which are associated with a lower risk of infection and post-operative hernia. The resulting shorter hospitalization time may lead to a reduction of the treatment costs [11].

\section{Conclusions}

We described a case of a very rare disease location which can be successfully treated laparoscopically with all of its advantages for the patient. The LUS as a method of intraoperative guidance during difficult and hazardous preparation navigated the surgeon in the hostile environment and enabled successful performance of the procedure. The LUS enabled the surgeon to find the right plane of dissection without injury to the surrounding tissues.

\section{Conflict of interest}

The authors declare no conflict of interest.

\section{References}

1. Hilton DA, Hanemann CO. Schwannomas and their pathogenesis. Brain Pathol 2014; 24: 205-20.

2. Yin SY, Zhai ZL, Ren KW, et al. Porta hepatic schwannoma: case report and a 30-year review of the literature yielding 15 cases. World J Surg Oncol 2016; 14: 103.

3. Lee WH, Kim TH, You SS, et al. Benign schwannoma of the liver: a case report. J Korean Med Sci 2008; 23: 727-30.

4. Cohen LM, Schwartz AM, Rockoff SD. Benign schwannomas: pathologic basis for CT inhomogeneities. AJR Am J Roentgenol 1986; 147: 141-3.
5. Miettinen M, Lasota J. Gastrointestinal stromal tumors: review on morphology, molecular pathology, prognosis, and differential diagnosis. Arch Pathol Lab Med 2006; 130: 1466-78.

6. Fonseca GM, Montagnini AL, Rocha Mde S, et al. Biliary tract schwannoma: a rare cause of obstructive jaundice in a young patient. World J Gastroenterol 2012; 18: 5305-8.

7. Wada Y, Jimi A, Nakashima O, et al. Schwannoma of the liver: report of two surgical cases. Pathol Int 1998; 48: 611-7.

8. Momtahen AJ, Akduman El, Balci NC, et al. Liver schwannoma: findings on MRI. Magn Reson Imaging 2008; 26: 1442-5.

9. Hariharan D, Constantinides V, Kocher HM, et al. The role of laparoscopy and laparoscopic ultrasound in the preoperative staging of patients with resectable colorectal liver metastases: a meta-analysis. Am J Surg 2012; 204: 84-92.

10. Russolillo N, D’Eletto M, Langella S, et al. Role of laparoscopic ultrasound during diagnostic laparoscopy for proximal biliary cancers: a single series of 100 patients. Surg Endosc 2016; 30: 1212-8.

11. Śmigielski JA, Piskorz $七$, Koptas W. Comparison of treatment costs of laparoscopic and open surgery. Videosurgery Miniinv 2015; 10: 437-41.

Received: 13.04.2017, accepted: 17.05.2017. 